\title{
How much exercise do we need to improve our immune system?: second series of scientific evidence
}

\author{
Yong-Seok Jee (iD https://orcid.org/0000-0001-6797-0843
}

Exercise has been called an antigen, since exercise has been identified as a behavioral factor that can improve immune function and serve as a form of vaccination against certain diseases or infections (Jee, 2019). The author from this study calls it "exercise antigen." Exercise antigen has a protective effect on the human body when the appropriate amount is supplied. However, the appropriate amount of exercise has not yet been established, and the opinions are divided among various researchers.

Many studies have been conducted to investigate the relationship between exercise and the body's immune response. Exercise has been identified as a behavioral factor that can boost immune function in some settings and therefore potentially serve as an adjuvant for immune responses. Exercise-induced changes in immune function can be viewed between acute exercise and chronic exercise training. Acute exercise refers to a single bout of exercise, while chronic exercise refers to an extended period and frequency of exercise. Many studies have reported a sudden and temporary change in the immune system after a single bout of exercise, which disappears shortly afterwards. On the other hand, exercise that is done consistently over a longer period of time results in positive or negative adaptations to the immune system. Such responses and changes depend on exercise intensity and duration for both acute and chronic exercise. If the exercise intensity is too weak, or the duration is too short, it will be ineffective to act as an exercise antigen. Conversely, exercising with too high of an intensity or too long of a duration can act as toxins, which results in cell damage and destruction.

In this editorial, the author will divide the section on exercise and immunity into several parts and provide useful information for prevention and rehabilitation. The first part will address the immune system's response to acute exercise. Acute exercise is known to have many short-term effects on immune function, but there appear to be contrasting effects of moderate exercise bouts and prolonged/intense exercise bouts. At the beginning of exercise, homeostasis is disrupted and various neuroendocrine, metabolites and immune responses are induced in proportion to exercise intensity and exercise duration. It is well known in the academic world that leukocytes, T cells, B cells, Natural killer cells, immunoglobulins, and cytokines, which are constantly changing during and after exercise, can seriously affect the body's resistance to disease. Peake et al. (2005) stated that exercise induction of a pro-inflammatory environment in the muscles, especially in the case of muscle-damaging exercise, may result in increased lymphocyte homing to the site of vaccine administration, and/or enhanced antigen uptake and processing, making the initial phase of the immune response more efficient. Campbell et al. (2009) reported that exercise has been shown to preferentially mobilize leukocytes with tissue-homing potential that contribute to the pro-inflammatory milieu. Leukocytosis, resulting from acute exercise, is driven by neuroendocrine substances and increases the circulation of monocytes and dendritic cells (Ho et al., 2001). These are potential antigens that increase the likelihood of migration to the site of antigen exposure. Finally, lymph drainage is known to be elevated by muscular contractions and thus, exercise may enhance immune cell transport from the site of antigen administration to the drainage of lymph nodes. The measurement of the vaccination response can be quantified in two main ways: the plasma cells' production of antibodies and the response of memory lymphocytes that stim- 
ulate antigens.

At present, there are many infectious diseases caused by viruses or bacteria, causing harm to many people. At this point, it is a priority for the Journal of Exercise Rebabilitation to further study what kind of exercises are best, as well as how individuals should exercise.

*First series is presented in J Exerc Rehabil 2019;15(3):339340 .

\section{CONFLICT OF INTEREST}

No potential conflict of interest relevant to this article was reported.

\section{REFERENCES}

Campbell JP, Riddell NE, Burns VE, Turner M, van Zanten JJ, Drayson
MT, Bosch JA. Acute exercise mobilises CD8+ T lymphocytes exhibiting an effector-memory phenotype. Brain Behav Immun 2009;23:767775 .

Ho CS, López JA, Vuckovic S, Pyke CM, Hockey RL, Hart DN. Surgical and physical stress increases circulating blood dendritic cell counts independently of monocyte counts. Blood 2001;98:140-145.

Jee YS. Exercise is an antigen for vaccination: first series of scientific evidence. J Exerc Rehabil 2019;15:339-340.

Peake J, Nosaka K, Suzuki K. Characterization of inflammatory responses to eccentric exercise in humans. Exerc Immunol Rev 2005;11:64-85.

Research Institute of Sports and Industry Science, Hanseo University 46 Hanseo 1-ro, Haemimyeon, Seosan 31962, Korea E-mail: jeeys@hanseo.ac.kr 